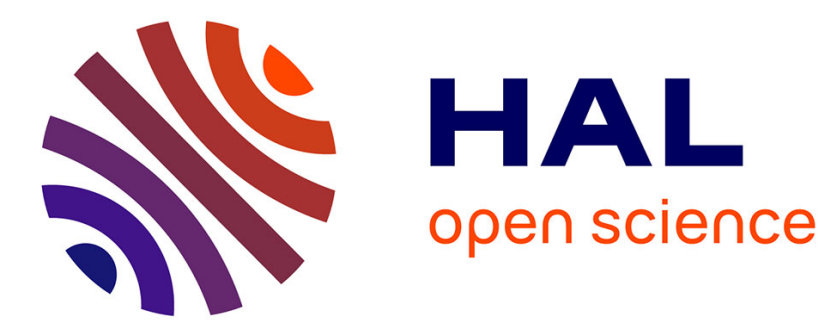

\title{
Meyerson critique ou héritier de Comte?
}

Bernadette Bensaude-Vincent

\section{To cite this version:}

Bernadette Bensaude-Vincent. Meyerson critique ou héritier de Comte?. Dialogue: Canadian Philosophical Review / Revue canadienne de philosophie, 2008, XLVII, pp.3-23. 10.1017/S0012217300002353 . halshs-00350690

\section{HAL Id: halshs-00350690 https://shs.hal.science/halshs-00350690}

Submitted on 22 Jan 2009

HAL is a multi-disciplinary open access archive for the deposit and dissemination of scientific research documents, whether they are published or not. The documents may come from teaching and research institutions in France or abroad, or from public or private research centers.
L'archive ouverte pluridisciplinaire HAL, est destinée au dépôt et à la diffusion de documents scientifiques de niveau recherche, publiés ou non, émanant des établissements d'enseignement et de recherche français ou étrangers, des laboratoires publics ou privés. 


\title{
Articles
}

\section{Meyerson critique ou héritier de Comte?}

\author{
BERNADETTE BENSAUDE-VINCENT Université Paris X-Nanterre
}

RÉSUMÉ : Émile Meyerson est, à juste titre, perçu comme un adversaire du positivisme d'Auguste Comte, mais une analyse de quelques passages de ses auvres étayée par sa correspondance montre combien est ambivalente sa relation à Comte. Nous proposons de poursuivre les réflexions qu'ébauche Meyerson sur sa relation à Comte, pour repenser la notion d'influence, trop souvent perçue comme un flux d'idées passivement reçues et plus ou moins digérées. On montrera que l'œuvre de Comte fut pour Meyerson une sorte de matériau d'idées auquel il fait subir un traitement, tout comme un industriel transforme des matières premières pour élaborer un produit fini.

ABSTRACT: Even though Émile Meyerson is rightly seen as an opponent of Comte's positivism, analyzing passages of his works with the help of his correspondence shows the ambiguity of his relation to Comte's philosophy. Drawing on Meyerson's remarks about his relation to Comte's philosophy, this article offers a new perspective on the notion of influence, which is too often perceived as passive or unassimilated reception of a stream of ideas. I argue that Meyerson treated Comte's ideas as a sort of raw matter he transformed into a finished product.

Dialogue XLVII (2008), 3-23

(C) 2008 Canadian Philosophical Association/Association canadienne de philosophie 


\section{Une œuvre de combat contre le positivisme}

Dans l'histoire de la philosophie, l'œuvre d'Émile Meyerson est, à juste titre, signalée comme une œuvre de combat contre le positivisme. Dès son premier ouvrage Identité et réalité paru en 1908, Meyerson adopte une position diamétralement opposée à celles du positivisme sur toutes les grandes questions de philosophie scientifique. C'est d'ailleurs sous forme de critique ouverte du positivisme comtien qu'il expose ses grandes thèses épistémologiques dans son second ouvrage De l'explication dans les sciences paru en 1921. Comte caractérise la science positive comme abstention de toute métaphysique. Meyerson proclame que tout savant fait de la métaphysique comme il respire. Comte définit la science positive comme recherche des lois et renoncement aux causes premières et ultimes. Meyerson défend le causalisme contre le légalisme. Comte estime que le but de la science positive est «savoir pour prévoir», que la curiosité est vaine et doit être régulée par l'action. Et Meyerson d'affirmer que la science n'a d'autre moteur ni d'autre but que de satisfaire la curiosité et de répondre aux besoins de notre intellect.

Une fois identifiés ces trois points de clivage, on pourrait procéder à un face à face des écrits des deux auteurs. En suivant ce programme, on montrerait que certaines critiques de Meyerson portent à faux car elles reposent sur une lecture biaisée des écrits de Comte. L'entreprise est classique et serait relativement aisée. De fait, Meyerson lui-même fut assez avisé pour s'apercevoir de l'ambivalence de son combat contre Comte et repérer ce qu'il lui a emprunté, tout en le critiquant. Sa clairvoyance nous oblige à aller plus loin que le simple face à face et rend notre programme beaucoup plus intéressant. Il ne s'agit plus d'arbitrer un duel entre deux philosophes en s'octroyant une position d'historien en surplomb. Il s'agit bien plutôt de poursuivre les réflexions qu'ébauche Meyerson sur sa relation à Comte pour repenser la notion d'influence, trop souvent perçue comme un flux d'idées passivement reçues et plus ou moins digérées. On montrera que l'œuvre de Comte fut pour Meyerson une sorte de matériau d'idées auquel il fait subir un traitement tout comme un industriel transforme des matières premières, pour élaborer un produit fini. Et du coup, cette étude de cas fournit un terrain idéal pour réfléchir sur ce que signifie hériter d'une tradition.

\section{Une influence avouée}

Partons d'une lettre autobiographique à l'essayiste Félicien Challaye, où Meyerson reconstruit son itinéraire philosophique. Il évoque d'abord ses études de chimie en Allemagne et signale l'influence du professeur Hermann Kopp, chimiste et historien - influence indirecte et à retardement puisqu'il a lu ses œuvres plus tard, après son installation en France, dans les bibliothèques parisiennes. Ensuite Meyerson mentionne l'œuvre de Comte. 
Mais bien plus forte encore et véritablement dominante fut celle [l'influence] d'Auguste Comte. Cette affirmation aussi peut paraître paradoxale. Car si j'ai constamment cité Comte, c'est presque toujours pour le combattre. Mais on peut observer, je crois, qu'un philosophe, en général, dépend le plus étroitement de ceux qu'il prétend réfuter. [...] Quant à moi, j'étais, par l'influence du milieu et même avant d'avoir lu une ligne de Comte, un pur scientifique selon la formule positiviste. Tout mon effort ultérieur n'a consisté qu'à me libérer de cette emprise, que je sens encore très forte en moi à l'heure actuelle. Et je me rends compte aussi que mon œuvre eût été impossible si celle de Comte ne l'avait précédée. C'est parce que Comte a eu le formidable culot (vous saisissez bien que c'est une expression admirative), à l'encontre de ce que nous sentons tous, que l'homme n'a pas besoin de se soucier de l'essence des choses et que la science peut donc se passer de métaphysique, et qu'il a essayé, au moins vaguement, de constituer une science ainsi faite, que j'ai pu, à mon tour, montrer que c'était là une entreprise impossible et dégager les véritables principes sur lesquels repose le raisonnement scientifique. Et c'est aussi lui qui a énoncé clairement, comme je l'ai rapporté dans l'Avant-propos de mon premier livre, que la méthode la meilleure pour se rendre compte des voies suivies par la raison consistait à analyser les raisonnements de la science. Sans doute n'a-t-il suivi luimême ce programme que dans une faible mesure, mais il n'en est pas moins certain qu'à ce point de vue je puis me réclamer comme d'un ancêtre spirituel ${ }^{1}$.

Comment entendre cet aveu de dépendance? Meyerson évoque des emprunts dans la position du problème, mais suggère aussi quelque chose de plus diffus, plus difficile à saisir, quand il évoque le contexte dans lequel se déroule sa lecture de Comte. Il fait allusion à la reconversion qu'il a entreprise dans les années 1890. Étant établi à Paris en 1882, il fréquente le laboratoire de Paul Schutzenberger au Collège de France, puis exerce le métier d'ingénieur dans une fabrique de colorants chimiques. En 1889, il abandonne la carrière de chimiste et prend un emploi de rédacteur pour la politique étrangère à l'agence Havas, ce qui lui laisse quelque loisir et lui permet de publier des articles sur l'histoire de la chimie. On notera déjà un parallèle avec le parcours de Comte qui abandonna la carrière scientifique à laquelle le destinaient ses études à l'École polytechnique pour s'aventurer sur le terrain de l'écriture philosophique en devenant secrétaire de Saint Simon. Toutefois c'est l'histoire de la chimie, plutôt que les événements, qui attira Meyerson vers la philosophie ${ }^{2}$. Durant les années 1880, Meyerson lit avidement, dévore les bibliothèques et ces lectures constituent son éducation philosophique. Lecteur insatiable, Meyerson amasse au fil des années un bagage philosophique si lourd que l'érudition envahit l'espace de ses propres écrits. Si l'œuvre de Comte fut déterminante, c'est indéniablement parce que sa lecture fut l'une des premières choisies par ce chimiste-historien. Et en tant qu'historien, 
Meyerson juge sévèrement l'histoire des sciences pratiquée par Comte au fil des leçons du Cours de philosophie positive.

Dans son récit autobiographique, Meyerson associe sa découverte du positivisme français à un travail d'émancipation à l'égard de l'emprise scientifique. L'expression est forte. Même si on doit faire la part des déformations qu'impose toute reconstruction autobiographique, Meyerson présente la science comme une tutelle, qui exerce une emprise sur les esprits $^{3}$. Et il fait de Comte le représentant ou porte-parole de cette autorité spirituelle. Ainsi associe-t-il la critique de Comte aux motivations justifiant son abandon des sciences.

C'est en tenant compte de la situation et de la disposition d'esprit de Meyerson lecteur que l'on voudrait ici aborder la question des rapports entre Comte et Meyerson. Le but n'est pas de repérer des emprunts, ni de dresser une liste de contresens de lecture pour tester la fidélité ou les infidélités du lecteur, mais plutôt de repérer comment Meyerson s'approprie la pensée de Comte pour forger ses propres thèses. La lecture de Comte a fourni à Meyerson des clés d'interprétation et d'analyse, elle lui a permis de rationaliser son propre cheminement, en lui donnant du recul et de la hauteur par rapport à ses pratiques quotidiennes du laboratoire ou de l'usine. Dès lors, notre programme est tout tracé : tenter de saisir comment Meyerson capture et canalise l'œuvre de Comte afin de façonner son propre programme. Et en inversant l'ordre des arguments avancés par Meyerson dans le passage ci-dessus où il évoque sa relation à Comte, nous commencerons par les questions de méthode avant d'aborder les questions de doctrine, conformément d'ailleurs à l'habitude prise par Comte dans le Cours de philosophie positive.

\section{Reconnaissance de dette}

Meyerson choisit d'avouer sa dette à l'égard de Comte en ce qui concerne la méthode qu'il a suivie tout au long de son œuvre, à savoir une approche historique de la pensée scientifique. "C'est le mérite incontestable d'Auguste Comte d'avoir insisté sur le rôle que doit jouer [...] l'histoire des sciences» $(E S$, ch. 18, p. 849). Convaincu qu'«on ne connaît pas complètement une science tant qu'on n'en sait pas l'histoire $\rangle^{4}$ et que la philosophie des sciences séparée de leur histoire est tout à fait stérile, Comte a rompu avec l'approche classique en termes de facultés mentales mobilisées dans la démarche scientifique pour «analyser les produits de la pensée» scientifique.

Dès Identité et réalité, Meyerson est en possession de cette méthode qu'il caractérise dans la préface par un double détour : détour par l'analyse de la science pour rendre compte des processus de la pensée commune, et détour par la longue durée historique. La procédure doublement indirecte est référée à Comte : 
C'est à l'aide de l'histoire des sciences que nous chercherons la solution des problèmes concernant le sens commun. C'est un procédé qui a quelque analogie avec celui préconisé par Auguste Comte, et bien que nous ne songions pas à dénier comme ce dernier la possibilité de toute psychologie introspective et que, comme on le verra par la suite, les résultats auxquels nous parvenons soient très différents de ceux exposés par le fondateur du positivisme, nous croyons que son mérite a été grand de proclamer la fertilité de la méthode a posteriori pour la découverte des lois qui régissent l'esprit humain ${ }^{5}$.

Le passé doit être déployé dans toute son épaisseur, la science dépassée étant un terrain encore plus fertile que la science sanctionnée. Pour voir comment fonctionne la science, comment elle transforme un ensemble de sensations en une suite d'énoncés scientifiques, rien ne vaut la science périmée car elle autorise de la part de l'observateur le détachement qui donne accès à l'objectivité ( $E S$, ch. 18, p. 840). La dette n'est pas négligeable car c'est précisément par cette méthode, l'attention à la science périmée, que Meyerson a exercé une influence notoire sur les générations suivantes, notamment sur Hélène Metgzer, Alexandre Koyré et Thomas Kuhn ${ }^{6}$.

Cependant Meyerson donne lui-même des raisons de douter de la dette qu'il avoue à l'égard de Comte. En effet, la méthode historique, il ne l'a pas trouvée chez Comte, ni chez Erdmann, mais chez Hermann Kopp. D'après son récit autobiographique, son intention initiale était d'écrire une histoire de la chimie. Meyerson rapporte son glissement de l'histoire vers la philosophie des sciences, à une hypothèse qu'il dit avoir trouvée chez Kopp.

Tout de suite presque, la direction de mes recherches changea; je cessais de m'occuper de l'histoire de la chimie pour m'intéresser aux ressorts cachés de cette évolution. En effet, a priori en quelque sorte, s'était établie en moi cette conviction inébranlable qu'il devait y avoir, en tout savoir et sous quelque forme qu'il se présentât, un canevas unique, qu'il ne se pouvait pas, en dépit des apparences, que par exemple, la science du moyen-âge fût quelque chose d'essentiellement différent à ce point de vue de la science moderne. Cette conviction me venait-elle de Kopp? Cela se peut, car si je parcours maintenant un chapitre de sa Geschichte, j'y vois parfaitement sous-entendue cette idée que les alchimistes, les iatrochimistes et les phlogisticiens cherchaient, tout comme nous, l'explication des réactions par la notion de quelque chose qui persistait et qui était simplement censé changer de place; il faut cependant que cette conception y soit bien peu apparente, pour que personne, avant moi, ne l'y ait découverte. Et alors, je suis plutôt porté à supposer que cette idée m'est venue d'ailleurs (plus ou moins spontanément, si j'ose dire) et que je l'ai simplement retrouvée chez Kopp (lettre à Challaye, s.d., A 408/9). 
Qu'il l'ait piochée ou simplement retrouvée chez Kopp, l'idée d'un «canevas unique» émerge d'une approche historique des sciences. De plus, Meyerson avoue une deuxième source d'influence, Charles Renouvier, qu'il lisait à la Bibliothèque nationale tout en amassant des notes pour son histoire de la chimie. C'est Renouvier, dit-il, qui lui a fait découvrir l'œuvre de Kant. Et Meyerson prend l'habitude de désigner l'étude historique des mécanismes de la pensée en termes kantiens comme «méthode a posteriori».

Dès lors, si Comte n'est que l'une des sources possibles de la méthode d'analyse caractéristique de Meyerson, quel est le sens de cette reconnaissance de dette? Et pourquoi est-elle assortie de critiques? Meyerson reproche à Comte de n'être pas assez comtien, de ne pas appliquer assez rigoureusement sa méthode historique. C'est donc bien qu'il a d'autres références, qu'il a appris cette méthode chez des auteurs plus exigeants que Comte, des auteurs qui ne se contentent pas d'un «coup d'œil» historique global comme Comte, et font des analyses fouillées, érudites, comme Kopp. On peut avancer l'hypothèse que si Meyerson paye ici un tribut à Comte pour un outil de travail qui lui vient d'ailleurs, c'est par souci de s'inscrire dans une lignée, de se doter d'un «ancêtre spirituel». Car la méthode historique caractérise globalement la philosophie des sciences qui se pratique en France à l'époque de Meyerson, de Paul Tannery à Gaston Bachelard en passant par Pierre Duhem, Léon Brunschvicg, Abel Rey et d'autres, moins célèbres ${ }^{7}$. Le choix de cette méthode a pour ainsi dire «naturalisé» l'œuvre de Meyerson en l'inscrivant dans une tradition et facilitant son intégration dans les milieux philosophiques français. Meyerson s'identifie si bien à cette tradition française que, lorsqu'il découvre le Cercle de Vienne au début des années 1930, il s’indigne que les positivistes aient tendance à oublier la source de leur philosophie : ni Mach, ni «les hommes du Wiener Kreis» ne citent Comte en parlant du positivisme (lettre à Lalande, s.d. [mars 1932], A/408, 60).

\section{L'«erreur» de Comte}

«La théorie positiviste repose au fond sur une erreur psychologique palpable : il n'est pas exact qu'en faisant de la science, nous ayons uniquement pour but l'action» (ES, ch. 2, p. 55). L'erreur de Comte, d'après Meyerson, est double : il dénie le rôle prépondérant de la curiosité naturelle; et il dénie que l'explication est une finalité de la science. C'est en termes d'erreur et avec des «constats» que Meyerson critique la théorie positiviste. Il dénonce des erreurs factuelles dans le positivisme et il lui oppose de prétendues évidences factuelles. Les premières sont empruntées au registre psychologique : «la soif de savoir» que «chacun de nous sent en luimême»; la satisfaction qu'on éprouve devant une explication. Pour illustrer ces «tendances naturelles» Meyerson invoque l'autorité des grands savants et cite des propos de Georges Cuvier, Claude Bernard, Louis Pas- 
teur, Henri Poincaré (ibid., p. 58-62). Il conclut que, sans cette tendance à rechercher les causes et le mode de production des phénomènes, l'histoire des sciences serait énigmatique. Ensuite il oppose à la définition comtienne de la science positive comme recherche des lois le «fait brutal» de l'existence de la science explicative. Ce fait est illustré par la pratique des savants les plus récents. En commentant longuement le Conseil Solvay de 1911, Meyerson montre que, avec leur «pourquoi» et leur «d'où vient», les Einstein et les Planck transgressent sans cesse les interdits comtiens.

Ce type d'argumentation fondé sur des «faits» revient à traiter le positivisme comme une théorie scientifique falsifiable par l'expérience, plutôt que comme un système philosophique. Et Meyerson laisse entendre que Comte (comme Hegel d'ailleurs) a eu l'audace de nier les faits (ibid., p. 71). Il est, de fait, assez difficile de cerner en précision la cible des critiques de Meyerson. Il s'attaque à «la doctrine positiviste». Il signale parfois des divergences entre Comte et «les positivistes d'aujourd'hui» mais sans les nommer (cf., par exemple, ibid., ch. 4, p. 145). Par moments, on a l'impression que la cible de ses critiques est plus Ernst Mach que Comte. En effet, Mach ne se contente pas de rejeter le concept de cause hors de la science, il lui substitue la notion de fonction; il affirme que les sciences de la nature décrivent mais n'expliquent pas, que les lois sont des tableaux ou résumés économiques, et surtout il rejette la notion de substance ou de chose que Comte n'a jamais explicitement attaquée ${ }^{8}$. Pourtant c'est bien le Cours de philosophie positive de Comte que Meyerson a lu et annoté dans toute son épaisseur et qu'il cite à maintes reprises. À l'occasion, il signale des divergences dans le mouvement positiviste, telle que la position de Littré, plutôt favorable à la finalité explicative de la science (ibid., ch. 2, p. 57). En lecteur assidu et commentateur minutieux, Meyerson mentionne à plusieurs reprises des nuances dans la pensée de Comte. Il n'ignore pas que Comte a déclaré que l'esprit humain a une curiosité spontanée pour les causes premières, que l'empirisme brut est impossible, etc., et d'autres thèses encore qu'il partage avec lui. Il estime que Comte avait un projet semblable au sien - décrire la pensée et le credo des scientifiques ${ }^{9}$ — et que ce projet s'est trouvé bridé, déformé par un système de pensée, dont Comte fut prisonnier. C'est ainsi qu'il interprète les limitations et interdits que Comte a voulu imposer aux recherches sur les astres, à l'application des statistiques, etc. Mais quel est ce carcan doctrinal dont Comte aurait été à la fois le héraut et la victime? C'est un peu un fantôme qui resurgit çà et là dans les critiques adressées à Comte. On peut en repérer deux empreintes.

D'une part, Meyerson en attribue la paternité à Francis Bacon. La formule comtienne - «science d'où prévoyance, prévoyance d'où action»provient, selon Meyerson, de Bacon qui a fait de l'action le but de la science et qui a fondé toute sa philosophie sur ce dogme (ibid., ch. 2, p. 55). L'empirisme de Bacon aurait pareillement conduit Comte à nier le rôle des théories, et à dénier l'effort rationnel pour n'exalter que la science expérimentale. 
D'autre part, Meyerson invoque un «esprit du temps», des années 1800 1830 , se traduisant par un manque de respect à l'égard de la science. C'est la raison qui justifie le rapprochement qu'il opère entre Hegel et Comte au chapitre 13 de L'explication dans les sciences. Malgré les différences manifestes de leur système, Hegel et Comte sont «fils de leur temps» en ce qu'ils traitent tous deux la science de leur époque avec une certaine désinvolture; tous deux prétendent la régir; tous deux «mutilent» la science en la réduisant à sa dimension expérimentale; tous deux méconnaissent - ou pire, interdisent - l'effort de la science vers la rationalité ${ }^{10}$.

Ainsi le positivisme qui est la cible des attaques de Meyerson semble être une tradition essentiellement caractérisée par un credo empiriste et un rejet de la rationalité à l'œuvre dans les sciences. C'est une sorte de main invisible qui, depuis Bacon, tirerait les ficelles de la science moderne en se renforçant au début XIX ${ }^{\mathrm{e}}$ siècle. Et Meyerson laisse entendre que les idées personnelles de Comte furent contraintes par le carcan de ce système, qui le conduisit à nier l'évidence en neutralisant les nuances de sa description des sciences. Comme on le verra plus loin, Meyerson va jusqu'à prétendre parfois qu'il comprend mieux le positivisme que Comte lui-même.

\section{Le «culot» de Comte}

Examinons maintenant la question de la relation entre science et métaphysique car c'est à la fois le point de désaccord le plus profond et le motif qui force l'admiration de Meyerson.

Comte a eu le formidable culot (vous saisissez bien que c'est une expression admirative), à l'encontre de ce que nous sentons tous, que l'homme n'a pas besoin de se soucier de l'essence des choses et que la science peut donc se passer de métaphysique (lettre à Challaye, s.d., A 408/9).

Voilà une remarque pour le moins étrange. Car l'idée que la science doit se tenir à l'écart de la métaphysique n'est certes pas la proposition la plus originale de Comte; on serait même tenté de dire qu'elle reprend un cliché répandu dans les traités de science. Meyerson présente donc comme paradoxale une thèse des plus banales, qui fait partie de la vulgate sur les sciences. En revanche, il faut un certain «culot» pour faire passer comme fait indiscutable le «besoin de se soucier de l'essence des choses» ${ }^{11}$. Enfin les deux thèses attribuées à Comte ne sont pas très comtiennes. Meyerson semble oublier que chez Comte «métaphysique» désigne un «état», une étape nécessaire de l'essor de l'esprit humain qui précède l'état positif et succède à l'état théologique. C'est pourquoi Comte reconnaît qu'il existe un «besoin de se soucier de l'essence des choses» mais veut s'en dégager. La tendance naturelle spontanée de l'esprit humain vers les questions absolues est une pièce maîtresse du positivisme comtien car elle est utile et bénéfique au commencement pour exercer et développer les facultés 
intellectuelles ${ }^{12}$. Quant à la possibilité pour la science de se passer de métaphysique, elle n'est pas non plus garantie dans le système comtien. Exclure les questions théologiques et métaphysiques est la condition pour accéder à la science positive, mais c'est un devoir, un impératif qui exige une ascèse. Il coûte tant d'efforts de renoncement que les plus grands héros fondateurs de la science positive - Descartes, Lavoisier, etc. — n'ont pas réussi à éliminer toute trace de métaphysique de leurs œuvres. Et Comte admet qu'il y a toujours des risques de «régression» car les savants tendent à «retomber» dans la métaphysique ${ }^{13}$. Force est donc d'avouer que la remarque de Meyerson n'a rien d'évident venant d'un lecteur tant soit peu attentif de Comte. En outre, elle présuppose au moins un postulat métaphysique déguisé en évidence factuelle qui porte sur l'existence d'un besoin irrépressible de métaphysique.

Il faut donc examiner de plus près les arguments de la critique du positivisme en se reportant, par exemple, au premier chapitre de L'explication dans les sciences. Meyerson y lance une première attaque frontale longuement et richement argumentée afin d'établir sa propre thèse — «la science exige le concept de chose» - qui donne son titre au chapitre. La critique commence par un témoignage d'admiration: le mérite de Comte est d'avoir fait du solide ce qui contraste avec le flou des discours de savants (ES, ch. 1, p. 22-23). Comte est un adversaire à la hauteur. L'enjeu de la dispute est l'abstention de toute métaphysique que Meyerson circonscrit en questionnant la thèse comtienne selon laquelle les hypothèses portent sur les lois et non sur le mode de production des phénomènes. Meyerson lui reconnaît une part de vérité. Oui, les savants cherchent des rapports, des règles. Mais, objecte Meyerson, ils ne peuvent s'empêcher d'édifier une métaphysique sui generis. D'une part, ils hypostasient les êtres hypothétiques - les éléments chimiques par exemple; d'autre part, alors que Comte refuse tout caractère transcendant aux lois, ils confèrent une portée objective à leurs règles ou lois comme si elles existaient dans la nature avant d'être découvertes (ibid., p. 34-36). Cette croyance à l'existence métaphysique des lois constitue, dans la terminologie de Meyerson, la «surcharge» des conceptions positivistes par les hommes de science (ibid., ch. 2 et 4, p. 122). Meyerson conclut que Comte n'a pas lui-même compris son système. S'il était conséquent, au lieu de déclarer que la science vise à établir des lois entre phénomènes (ce qui suppose des choses derrière les phénomènes) il aurait dû conclure qu'elle établit des lois entre des sensations. Meyerson sait bien que Comte ne fonde pas la science sur la sensation. Il n'ignore pas les critiques répétées de Comte contre l'empirisme baconien (cf. ibid., ch. 2, p. 71-72). C'est pourquoi il n'hésite pas à corriger Comte et prétend que John Stuart Mill aurait mieux compris le positivisme que Comte lui-même (ibid., ch. 1, p. 37).

Contre la conception positiviste (mais non comtienne) de la loi comme simple rapport entre sensations, Meyerson invoque d'abord «l'attitude 


\section{Dialogue}

mentale» des physiciens et le devenir de la thermodynamique - science abstraite fondée uniquement sur la généralisation de lois expérimentales dépassée par la théorie cinétique qui postule l'existence réelle des molécules et conduit à la mécanique statistique. D'où il tire deux conclusions : premièrement il n'y a pas de science vierge d'ontologie; deuxièmement, en déniant la métaphysique, on se fonde sur la métaphysique du sens commun au lieu d'élaborer une autre ontologie, plus durable, plus solide, propre à la construction scientifique. L'exemple maintes fois resservi, de l'électricien qui ne peut pas s'empêcher de croire au courant qui circule dans le fil, illustre la métaphysique du sens commun; tout comme la croyance aux électrons illustre l'ontologie des scientifiques. Ironie de la critique. Pour justifier son désaccord profond, Meyerson s'appuie sur une thèse chère à Comte : la continuité entre science et sens commun. Cette thèse partagée joue un rôle majeur dans la philosophie de Meyerson et sert de point d'orgue à ses deux premiers livres. Esquissée au chapitre 9 d'Identité et réalité, elle fonde son entreprise, qui consiste à étudier les processus de la pensée commune à travers l'histoire des sciences. Au chapitre 18 de L'explication dans les sciences, Meyerson reprend le thème : il affirme qu'il y a identité de nature de la pensée dans tous ses registres d'exercice, avec seulement une hiérarchie des degrés d'ontologie: la science est ontologique dans son départ (sens commun) et forge une ontologie plus durable à partir d'êtres hypothétiques plus durables que les sensations ${ }^{14}$.

$\mathrm{Au}$ bilan, cette argumentation sommairement résumée (car Meyerson affine au fil des digressions) met en relief plusieurs aspects surprenants. Premièrement, les désaccords patents entre Comte et lui-même se jouent sur fond d'une entente profonde quant au statut de la science par rapport à la connaissance commune, et au rejet de l'empirisme; deuxièmement, Meyerson refuse cependant de prendre au sérieux cette critique de l'empirisme chez Comte. Ce biais de lecture n'a pas échappé à l'attention de Lucien Lévy-Bruhl. Dans une lettre du 12 avril 1921 (A 408/65), où il le remercie pour l'envoi de L'explication dans les sciences, il souligne leur divergence :

J'interprète Comte un peu autrement que vous le faites : il est, selon moi, plus près de Descartes et moins près de Bacon qu'il ne semble d'après votre livre ou, si vous préférez, moins loin de vous-même que vous ne le croyez, et c'est une des raisons du succès de sa doctrine auprès d'un grand nombre de savants.

Troisièmement, la question métaphysique mobilise et subordonne tous les autres points de désaccord, notamment l'opposition entre le «légalisme» de Comte et le «causalisme» de Meyerson qui joue sur l'identification de la cause à la chose, sur l'affirmation qu'il n'y a pas de rapports sans supports. Quatrièmement, Meyerson ne tire pas ses arguments de l'ensemble de l'histoire des sciences mais de quelques épisodes récents, tels que la mécanique statistique qui supplante la thermodynamique. Ce faisant, il 
transforme les faits en droit. À l'en croire, la victoire de Boltzmann sur les énergétistes signe la défaite du positivisme. Cinquièmement enfin, l'argument-clé de Meyerson en faveur de l'ontologie sous-jacente à la science est l'affirmation de la foi inébranlable du physicien. Meyerson prête aux savants une foi robuste et naïve, «la foi du charbonnier» qui les conduit non seulement à postuler l'existence des entités que crée leur intellect — atomes, électrons, ions, etc. — mais aussi à postuler la conservation des substances qui disparaissent, tels que les éléments chimiques qui s'effacent dans le composé.

Sur quoi se fonde cette affirmation? Sur le vécu, sur un sentiment intime, immédiat qui relève de la psychologie du savant. Meyerson fait souvent appel au témoignage d'un chimiste français, Georges Urbain, qu'il dit avoir lu et médité (ES, ch. 15, p. 636). Dans Les disciplines d'une science, Urbain notait que tout chimiste croit à la réalité du sulfate de baryte, c'està-dire à un hypothétique substrat des propriétés observées ${ }^{15}$. Meyerson utilise cette remarque d'un illustre chimiste comme argument d'autorité. À l'occasion d'une lettre, il loue sa perspicacité et renchérit sur le propos d'Urbain.

Il a fallu un esprit profondément philosophique par essence pour penser à poser, à des chimistes, la question telle que vous l'aviez formulée. Leur étonnement le prouve, et c'est pourquoi aussi il me paraît étonnant que, vous inspirant uniquement de recherches de méthode, vous ayez mis le doigt directement sur le point sensible. Car le chimiste, évidemment, n'y pense jamais. Non parce qu'il ne croit pas au réel, mais au contraire parce qu'il y croit trop, parce qu'il lui semblerait absurde de laisser effleurer sa «foi robuste» (comme vous la qualifiez à juste titre) du doute le plus léger (lettre à Urbain, 14 février 1932, A 408/102).

L'appel à l'autorité d'un savant comme Urbain est de haute stratégie car ce chimiste est connu pour ses prises de position positivistes. Il incarne en quelque sorte ce que Meyerson aurait pu devenir, s'il n'avait pas déserté la carrière scientifique. Sa conception de l'atome comme une fiction, une œuvre d'art, ne semble pas gêner le réalisme de Meyerson. Ici comme dans sa lecture de Comte, il reste sourd, aveugle, aux passages qui ne cadrent pas avec son schéma. En tout cas, il est clair que cette foi robuste constitue le point de divergence majeur avec Comte. Et Meyerson précise aussi à l'occasion que c'est bien là son principal reproche à Comte.

On doit constater la manière de penser du chimiste de laboratoire, si brillamment précisée par vous, en tant que fait, fait incontestable et dont le théoricien de la pensée scientifique ne peut pas ne pas tenir compte. C'est parce qu'il ne l'a pas aperçu, que Comte - que je considère comme une des penseurs les plus authentiquement grands qui aient vécu — est, selon moi, tombé dans l'erreur (ibid.). 


\section{La thèse du déni}

Force est d'avouer que, sur un plan strictement logique, le réquisitoire de Meyerson contre le positivisme est bien faible. L'argumentation pêche à bien des égards : lecture biaisée de l'auteur critiqué; utilisation sélective de l'histoire; quant au «constat» d'un acte de foi chez les scientifiques, c'est un argument dogmatique totalement infalsifiable dès lors qu'il est assorti de l'argument de rétorsion : dénier la métaphysique, c'est encore exprimer une métaphysique. La foi du physicien est bien le maillon le plus faible et l'on comprend que Bachelard ait pris plaisir et peu de peine à critiquer impitoyablement le réalisme dogmatique et naïf de Meyerson ${ }^{16}$.

Comment un philosophe rompu à l'argumentation, et qui passe pour rationaliste, peut-il maintenir des affirmations aussi peu étayées? Qu'estce qui l'autorise en plus à contredire les savants qui déclarent s'abstenir de toute métaphysique?

On touche ici un point sensible de la philosophie de Meyerson, au double sens de vulnérable et de ressenti. Indubitablement, Meyerson s'appuie ici sur son expérience personnelle, son vécu intime de chimiste, rompu à la chimie organique. Il a été formé dans une époque où l'on enseignait les formules structurales en refusant de croire à la réalité des atomes et molécules. Les chimistes du XIX ${ }^{\mathrm{e}}$ siècle, en particulier ceux qui s'occupent des synthèses organiques, comme ce fut le cas de Meyerson, exploitent les avantages de la notation atomique pour écrire les formules des corps tout en proclamant qu'ils ne croient pas à l'existence des atomes. August von Kekulé, qui a fait l'hypothèse de la structure hexagonale de la molécule de benzène - base de la plupart des composés organiques synthétisés au $\mathrm{XIX}^{\mathrm{e}}$ siècle - est particulièrement représentatif. Atomiste convaincu dans sa pratique de chimiste, il rejette néanmoins hors de la chimie la question ontologique de la réalité des atomes.

The question whether atoms exist or not has but little significance in a chemical point of view : its discussion belongs rather to metaphysics. In chemistry we have to decide whether the assumption of atoms is an hypothesis adapted to the explanation of chemical phenomena. More especially have we to consider the question, whether a further development of the atomic hypothesis promises to advance our knowledge of the mechanism of chemical phenomena. I have no hesitation in saying that, from a philosophical point of view, I do not believe in the actual existence of atoms, taking the word in its literal signification of indivisible particles of matter. I rather expect that we shall some day find, for what we now call atoms, a mathematico-mechanical explanation, which will render an account of atomic weights, of atomicity, and of numerous other properties of so-called atoms. As a chemist, however, I regard the assumption of atoms, not only advisable, bus as absolutely necessary in chemistry ${ }^{17}$. 
Meyerson n'y croit pas, il n'arrive pas à prendre ces scrupules ontologiques au sérieux. Sa conviction intime, indéracinable, repose non sur la logique mais sur sa propre pratique de chimiste organicien. Comment manipuler des substances, faire la synthèse de colorants, concevoir de nouvelles molécules en général sans croire qu'elles existent? On peut certes douter de leur nature, se disputer sur leur structure et leur conformation, mais douter de leur réalité, cela semble impossible. C'est pourquoi Meyerson n'hésite pas à mettre en doute les déclarations de Kekulé qu'il juge totalement en contradiction avec les pratiques de synthèse chimique.

Il [Kekulé] formule quelques fois des réserves, mais il ne le fait, on le sent bien, que du bout des lèvres, pour la forme. Au fond, il croit dur comme fer à l'existence de ces atomes, de leurs molécules et de leurs liaisons et il les manie sans façon exactement comme si c'étaient des objets de la sensation du sens commun ${ }^{18}$.

Sa familiarité avec les organiciens a suggéré à Meyerson qu'il existe un abîme entre les discours épistémologiques des scientifiques et leur pratique réelle. «Les chimistes ont souvent, dans leurs déclarations théoriques, renié les principes qu'ils suivaient invariablement dans leur pratique» $(E S$, ch. 6, p. 270).

On le voit, la thèse du déni est scandaleuse à bien des égards. D'une part, elle introduit la foi dans la science. Il y a de la croyance - et une croyance des plus naïves - dans cette activité, fleuron de la raison humaine. D'autre part, elle jette un doute sur l'authenticité des déclarations épistémologiques des savants ${ }^{19}$. Meyerson a beau jeu de reprocher à Hegel et Comte d'avoir manqué de respect envers la science! Il s'expose, lui, au reproche de manquer de respect envers les savants. Meyerson les soupçonne finalement de mauvaise foi. Vers la fin de sa vie, il va même jusqu'à suggérer que l'attitude des chimistes est ce qui l'aurait poussé à abandonner la chimie :

En ce qui concerne la réalité de l'atome chimique, la foi du chimiste de laboratoire était, il y a vingt, trente, ou même quarante-cinq ans (c'est-à-dire à l'époque où tout jeune je suis entré dans ce domaine) aussi ferme qu'elle peut l'être. C'est même à vrai dire l'étonnement que me faisait cette divergence flagrante entre la conviction intime du chercheur et la conviction philosophique qu'il prétendait avoir adoptée, mais qu'il ne proférait que du bout des lèvres (c'est-à-dire sans qu'elle exerçât la moindre influence sur la marche de son travail) qui a été un des points de départ des réflexions qui m’ont amené à chercher une épistémologie nouvelle (lettre à Bertoud, 1925, A 408/11). 
Donc le positivisme n'est aux yeux de Meyerson qu'une façade. Une épistémologie qui reste au «bout des lèvres» sans imprégner vraiment la science telle qu'elle se fait.

\section{Héritier révolté}

Si l'on en croit Meyerson, c'est un sentiment de révolte contre le positivisme de façade dans les milieux scientifiques de son époque qui aurait motivé son entreprise philosophique. Même si cette version des faits procède d'une rationalisation après coup, on doit reconnaître qu'il entre quelque chose d'intime et de passionnel dans ses critiques du positivisme.

Mais il serait superficiel de croire que la révolte conduit au rejet. Paradoxalement, elle produit l'effet inverse. La croisade que Meyerson entreprend contre le positivisme le pousse à s'approprier la nature même des questions soulevées par Comte. Oui, c'est à juste titre que Meyerson reconnaît sa dette envers Comte, car cette dette va bien au-delà de l'emprunt de la méthode a posteriori d'analyse des sciences. Meyerson est en quelque sorte «obligé» par l'œuvre de Comte, prisonnier de son système, comme Comte lui-même le fut, d'après Meyerson, de la tradition baconienne.

On montrera cet effet paradoxal sur deux thèses : l'établissement des lois et la relation de la science au réel ${ }^{20}$. La philosophie de Meyerson s'organise, comme celle de Comte, autour de l'opposition loi/cause. Le problème est posé exactement dans les mêmes termes que chez Comte : lois ou causes, quelle est la finalité de la science? Héritier de cette question, Meyerson répond à l'inverse de Comte et dénonce son «erreur». La science vise les causes et seule une explication identifiant le divers peut satisfaire la tendance impérieuse de notre intellect à l'identification. Cela ne l'empêche pas de continuer à élaborer la question des lois, à pousser plus loin l'analyse de Comte en lui donnant un tour plus kantien. À quelles conditions peut-on établir des lois de la nature? Critiquant ou assimilant des aspects de la philosophie de Comte Meyerson traite la question sous deux angles, du côté du sujet et du côté de l'objet.

Du côté du sujet, de l'activité de l'intellect, l'établissement des lois présuppose des théories et la tendance à l'identification. Sa réponse se confond donc avec une critique de l'empirisme baconien, que Meyerson s'obstine à ne pas voir chez Comte. Comte admettait, cependant, que l'observation - préalable à toute loi — exigeait des conceptions théoriques, et affirmait par conséquent que l'empirisme était impossible ${ }^{21}$. Meyerson formule des critiques assez voisines et parallèles sur l'impossibilité d'induire des lois à partir des seuls faits, tout en persistant à faire l'amalgame entre Bacon et Comte (ES, ch. 3, p. 113 et ch. 15, p. 592-93). Et quand parfois il consent à dire que Comte reconnaît un rôle à la rationalité, il lui reproche, comme à Bacon, le côté machinal de la démarche de découverte des lois. 
Quant aux conditions objectives — du côté de l'objet - Meyerson reconnaît la justesse de la remarque de Comte sur la nécessité d'une conformité entre la raison et la nature, mais désapprouve aussitôt les doutes de Comte sur la possibilité de maintenir les lois de la nature en cas d'investigation détaillée (ibid., ch. 4). Meyerson semble convaincu que les lois générales peuvent résister à toute épreuve et reproche à Comte d'avoir condamné l'usage des instruments de haute précision (ibid., p. 123).

Meyerson ne se détache de Comte sur la question des lois qu'après sa lecture d'Arthur James Balfour. À ce politicien philosophe (avec qui il échange des vues non seulement sur la philosophie mais aussi sur la situation de la Palestine), Meyerson emprunte le concept de «structure fibreuse». Cette métaphore suggère que la nature est tissée de liens ou relations enchevêtrées; tous les phénomènes sont donc interdépendants, mais en isolant une fibre on parvient à saisir parfois des îlots de cohérence.

Ces séries de phénomènes cohérents dont nous venons de parler ressemblent en effet aux fibres d'un tissu organique, qui ne sont sans doute pas entièrement indépendantes de ce tissu, auquel elles adhèrent du reste fortement aux deux bouts, mais dont, entre ces deux points, les attaches sont légères et en petit nombre, de sorte que la fibre peut, au moins grosso modo, être isolée (ibid., p. 111).

Si notre recherche des lois ou relations consiste à tirer localement une fibre avec quelques autres voisines, nous ne parvenons jamais à dénouer la pelote entière des relations. Meyerson tire de sa lecture de Balfour les conditions de possibilité pour l'établissement des lois. Premièrement, la légalité suppose la répétition, c'est-à-dire l'existence de phénomènes réguliers. Or, il n'y a jamais de répétition au sens strict : tout est événement, la régularité est introduite dans la nature par l'intellect. Ce premier temps suggère que les lois sont des constructions intellectuelles, des artefacts introduits dans une nature rebelle. Meyerson verserait-il dans l'idéalisme ou le constructivisme? Pas vraiment, car dans un deuxième temps, il admet que si l'entendement parvient à saisir des lois, c'est parce que dans la pelote d'interactions entre phénomènes, il existe une hiérarchie, avec des relations de dépendance à sens unique ${ }^{22}$. Or cette dépendance des phénomènes est l'un des fondements objectifs de la hiérarchie des sciences, établie par Comte. De l'astronomie, à la physique, à la chimie, puis à la biologie, et à la sociologie il y a dépendance croissante des phénomènes, d'où leur complexité croissante (Comte, Cours, t. 1, leçon 2, p. 54-58). Meyerson redécouvre donc par d'autres voies des thèses essentielles du positivisme comtien.

Meyerson réintègre pareillement et par des voies détournées des thèses de Comte sur la question fondamentale de la finalité de la science. Après avoir attaqué de front la thèse comtienne qui voue la science positive à dire les rapports entre phénomènes au lieu de chercher à saisir l'essence du 
réel, il avoue cependant lui aussi que la science est impuissante à pénétrer le réel.

Science et métaphysique poursuivent toutes les deux le même but, la connaissance du réel. Comte l'a vu, mais il a certainement eu tort, le but utilitaire qu'il a assigné à la science n'est que secondaire, celui auquel le savant obéit en premier lieu étant la curiosité, la soif de savoir, de connaître le fond des choses. Le point de départ est également le même : il est dans laperception, en d'autres termes dans la réalité du sens commun. La différence n'est que dans les voies suivies. Le physicien cherche d'abord, par l'observation, par l'expérimentation, à multiplier les rapports entre les choses du monde extérieur, alors que le philosophe, en analysant directement la perception, cherche à déterminer, dans celle-ci, la part attribuable à l'action de l'intellect, afin d'arriver ainsi, par soustraction, à connaître l'être en soi. Il est clair d'ailleurs que ce mode de procéder de la philosophie est le seul bon, que celui de la science ne peut mener à rien (au point de vue de la connaissance du réel, s'entend). En effet, il est absurde de supposer, comme le fait implicitement l'empirisme, que l'observation ou l'expérience puissent directement et pour ainsi dire machinalement nous apprendre quelque chose. Mais si nous voulons nous borner à un savoir purement empirique, il nous faut abstraire des lois et, pour ce faire, établir une hiérarchie des conditions du phénomène (puisque aussi bien des conditions absolument identiques ne se reproduisent jamais); en un mot, il nous faut raisonner. Donc, la science conserve constamment le facteur qu'il s'agit d'éliminer, et de ce chef, elle est impuissante à nous renseigner sur la nature du réel (lettre à Bergson, s.d., A 408/4).

L'argumentation ébauchée dans cette lettre à Bergson prend tout son sens à la lumière du chapitre sur le paradoxe épistémologique dans L'explication dans les sciences. Le chapitre 17 débute par une interrogation assez naïve sur la concurrence entre sa propre théorie de la science (tellement simple et évidente) et la théorie positiviste.

Comment se fait-il [...] que cette théorie ait surgi si tard et qu'à l'heure actuelle encore elle ait tant de peine à se faire agréer, au point que la conception positiviste de la science, si étriquée, expliquant si mal ce qui fait vraiment l'essence de la recherche scientifique, soit le plus souvent exposée de manière à la faire apparaître comme la seule possible? (ES, §17, p. 783)

Pour rendre compte du prestige de la doctrine positiviste, Meyerson propose un long détour par l'histoire de l'atomisme visant à montrer que la science moderne n'est pas en rupture avec la science antique car elle traduit toujours la même "structure intime» de notre pensée, à savoir la tendance à chercher l'identique sous le divers. Puis il démontre que les principes de conservation ne sont pas des lois empiriques, comme voudrait 
le faire croire le positivisme, mais des principes a priori, qui expriment la tendance de l'intellect à nier le changement. Meyerson en vient alors à dire que la science n'est pas seulement explicative mais aussi prédictive et pratique. Et ce n'est pas juste une concession au positivisme. La dualité explication/prévision est essentielle. Elle constitue le paradoxe fondamental de la science. Dans son désir de tout réduire à l'identique la raison aboutirait à la négation du réel, à une pure idée sans réalité, si la science n'était en même temps, la recherche des lois. Car les lois empiriques inspirent de nouvelles théories qui font découvrir de nouveaux faits expérimentaux et font ainsi avancer la science.

Or cette union entre la science des théories et celle des prévisions ou, si l'on veut, ce double aspect de la science (qui est véritablement une) crée, au point de vue qui nous occupe, cette situation étrange : que, pour expliquer, nous tendons à nier les phénomènes alors que, pour nous guider à travers le dédale qu'ils forment, nous devons, au contraire maintenir leur réalité (ibid., p. 824).

Le savoir expérimental est donc fondamentalement contradictoire. Tel est le message essentiel de Meyerson. Comte avait bien reconnu cette contradiction nuisible à l'«harmonie de l'esprit positif». Mais il considérait que l'«aveugle instinct de liaison», la tendance à réduire le divers à l'unité était une habitude contractée sous l'emprise de l'esprit théologique et que l'étude du monde extérieur devait en libérer les esprits positifs ${ }^{23}$. Meyerson, lui, ne considère pas cette tendance inhérente à la rationalité comme un résidu dont l'influence pourrait s'effacer. Et il n'espère pas davantage réduire la dualité grâce à une subtile dialectique entre les deux faces de la science. La solution dialectique a été écartée au fil des chapitres de critique de Hegel qui est, après Comte, le deuxième pôle de référence permettant à Meyerson de préciser sa philosophie de l'intellect. La contradiction inhérente au savoir expérimental est «flagrante et irrémédiable», car elle répond à une contradiction symétrique dans la nature qui est à la fois autre, extérieure à nous et produit de nos sensations, comme de notre raison. L'intelligence a besoin pour fonctionner de transporter ce conflit à l'intérieur d'elle-même (ibid., ch. 18, p. 827).

Cette conception très paradoxale de la science atténue la sévérité des jugements de Meyerson à l'égard de Comte, qui deviennent de plus en plus nuancés. Après l'exposé du paradoxe épistémologique, il est en mesure d'expliquer le prestige du positivisme dans les milieux scientifiques. Il rend compte du «sentiment intime du physicien obligé de réagir constamment contre ses propres propensions» aux théories chimériques.

Sans doute est-ce à tort et d'ailleurs vainement que le positivisme déclare la guerre à ce penchant auque l'homme - fût-il savant — est obligé de satisfaire; mais Comte a eu le mérite de prémunir le chercheur conte le danger d'un excès 
dans cet ordre d'idées, danger qui, en effet, le guette sans cesse. Et c'est sans doute le sentiment de cette situation qui a grandement contribué à assurer le succès éclatant de la doctrine, si manifestement insuffisante à d'autres égards (ibid., p. 836).

On voit donc que la présentation courante de Meyerson comme un antipositiviste n'est que partiellement adéquate, car il a construit sa propre pensée dans un rapport très ambivalent au positivisme. En même temps, cette ambivalence révèle la multiplicité des figures du positivisme au début $\mathrm{du} \mathrm{XX}^{\mathrm{e}}$ siècle.

Meyerson a certes élaboré son œuvre dans un geste de révolte contre un certain positivisme dominant dans les milieux scientifiques desquels il est issu. Il se révolte contre une doctrine qui règne en maître dans les discours des savants, parce qu'il juge que leur pratique dément leurs discours. Et cette révolte aurait, d'après les récits autobiographiques de Meyerson, déterminé sa vocation philosophique. Le positivisme est donc pour Meyerson quelque chose comme une «imposture intellectuelle». Ce positivisme ambiant biaise la lecture de Comte par Meyerson. Il attribue au fondateur $\mathrm{du}$ positivisme des thèses empiristes baconiennes sans cesse combattues par Comte. Mais on ne saurait parler d'un rejet du positivisme car Meyerson s'est imprégné de la philosophie de Comte au point d'élaborer sa propre épistémologie en fonction des thèses qu'il attribue à Comte. La catégorie d'influence n'est guère plus adéquate que celle de rejet car elle suggère une action à sens unique. Meyerson est allé chercher chez Comte de quoi nourrir sa philosophie. C'est plutôt en termes d'appropriation qu'il faudrait décrire sa relation à Comte. Meyerson s'approprie l'œuvre de Comte au sens où il assimile (suivant la tendance à l'identification) le projet comtien à son propre projet de décrire le fonctionnement de l'intellect humain. Le positivisme est une face de la science, constamment affrontée à l'appétit d'identification de la raison. La tendance métaphysique, dont Comte a reconnu la prégnance à l'âge métaphysique mais qu'il croyait pouvoir dépasser, est indépassable. Pour Meyerson, elle constitue l'un des pôles de l'intellect humain et c'est elle qui crée la tension nécessaire à l'activité scientifique.

On dira donc que Meyerson s'est posé en héritier de Comte, au sens où hériter, c'est recueillir un patrimoine pour s'en rendre propriétaire, en jouir et le transformer. Meyerson incorpore l'œuvre de Comte à sa propre entreprise philosophique et la perpétue dans une généalogie philosophique sans pour autant s'astreindre à la fidélité. En assumant cet héritage, il s'est inscrit dans une lignée française de philosophes des sciences. Par son infidélité même à celui dont il assume la succession, il crée la continuité d'une tradition de pensée. 


\section{Notes}

1 Brouillon de lettre de Meyerson à F. Challaye (sans date); Central Zionist Archives, Jérusalem, Fonds Meyerson A 408/9. La correspondance de Meyerson sera désormais citée de la manière suivante : lettre à Challaye, s.d., A 408/9.

2 Son ambition était d'écrire une histoire de la chimie alliant la clarté des Leçons de philosophie chimique de Jean Baptiste Dumas à l'érudition de la Geschichte der Chemie de Hermann Kopp (cf. note précédente).

3 Meyerson souligne combien même dans une position de «détachement philosophique» il est difficile de ne pas croire à la vérité de la science contemporaine (De l'explication dans les sciences [1922], Paris, Fayard, 1995, p. 843; désormais : $E S$ ). Cette idée d'emprise sur les esprits est l'une des dimensions que Thomas Kuhn — qui fut lecteur de Meyerson — prête au paradigme.

4 Comte, Cours de philosophie positive, 1830-42, t. 1, leçon 2, Paris, Hermann, 1975, p. 53 (désormais : Cours).

5 Identité et Réalité [1908], Paris, Alcan, 1912, p. xvi (désormais : IR).

6 Koyré reconnaît publiquement sa dette à l'occasion du centième anniversaire de la naissance de Meyerson (voir Bulletin de la Société française de philosophie, Commémoration du Centenaire de la naissance de deux épistémologues français : Émile Meyerson et Gaston Milhaud, Séance du 26 novembre 1960, avril 1961, p. 115-116). Metzger reconnaît son influence tout en se défendant d'être disciple de Meyerson (voir «La Philosophie d'É. Meyerson et l'histoire des sciences», Archeion, vol. 11, 1929, p. 32-42; reprint dans La méthode historique en histoire des sciences, Fayard, Paris, 1987, p. 95-106). Kuhn recommande les pratiques historiennes de Meyerson tout en désavouant sa philosophie (The Essential Tension, Chicago, University of Chicago Press, 1977, p. 11).

7 Michel Bitbol et Jean Gayon, dir., L'épistémologie française 1830-1970, Paris, Presses universitaires de France, 2006, p. 113-195. Voir aussi Anastasios Brenner, Les origines françaises de la philosophie des sciences, Paris, Presses universitaires de France, 2003.

8 Voir E. Mach, Erkenntnis und Irrtum, Lepzig, Barth, 1905 (trad. Marcel Dufour, La connaissance et l'erreur, Paris Flammarion, 1908, p. 275); Guillaume Garreta, «Ernst Mach, l'épistémologie comme histoire naturelle de la science», dans P. Wagner, dir., Les philosophes et la science, Paris, Gallimard, 2002, p. 624-658.

9 Voir, par exemple, ES (ch. 5, p. 213) : «Nul n'a été d'ailleurs, plus explicite à cet égard qu'Auguste Comte dans les moments où, ce qui lui arrivait quelquefois, comme nous l'avons vu, il perdait de vue sa doctrine et se contentait de formuler le credo scientifique de son époque».

10 Meyerson reconnaît toutefois une différence importante dans l'impact de leur œuvre sur les milieux scientifiques : alors que Hegel n'a suscité que mépris et dédain de la part des savants, Comte a eu une réelle influence.

11 Le reproche d'arbitraire et d'affirmation invérifiable a d'ailleurs été adressé à Meyerson par Gaston Milhaud dans sa recension de la seconde édition 
22 Dialogue

d'Identité et réalité (cf. «Une théorie récente de la causalité», La Revue du mois, vol. 14, no 83, 10 novembre 1912, p. 541-562).

12 Voir par exemple la présentation de l'état théologique dans le Discours sur l'esprit positif [1844], §7, Vrin, Paris, 1995, p. 49 (désormais : Discours).

13 Voir, par exemple, Cours, t. 2, leçon 56, p. 567 et 577-581; Discours, §15, p. $72-$ 73.

14 Les atomes sont les entités paradigmatiques de cette ontologie scientifique qui est à la fois inconsciente et générale au sens où elle crée du consensus (cf. ES, ch. 15, p. 686-687). Meyerson semble oublier que sur les atomes, Comte serait d'accord. Dans la $37^{\mathrm{e}}$ leçon du Cours de philosophie positive, il présente le principe général de l'atomisme comme une évidence non problématique et admise par tous (Cours, t. 1, p. 611; voir aussi B. Bensaude-Vincent, «Le positivisme fait-il obstacle au progrès scientifique?», dans A. Despy-Meyer et D. Devriese, dir., Positivismes, Bruxelles, Brepols, 1999, p. 217-244).

15 Georges Urbain, Les disciplines d'une science, la chimie, Paris, Libraire O. Doin, 1921, p. 18 (Meyerson cite ce texte d'après un article paru dans $L a$ grande revue en 1920).

16 Voir, par exemple, le chapitre «Le matérialisme composé», dans Gaston Bachelard, Le matérialisme rationnel, Paris, Vrin, 1953, p. 112-153.

17 A. Kekulé, «On Some Points of Chemical Philosophy», The Laboratory, I, July 27, 1867. Cité par Britta Görs, «Vom imaginären Atom zum räumlichen Gebilde : Der pragmatische Umgang mit dem chemischen Atomismus in der zweiten Hälfte des 19. Jahrhunderts», dans A. Jobmann, et B. Spinder, dir., Theorien über Theorien über Theorien, IWT-Paper Nr 24, Bielefeld University, 1999, p. 37-43.

18 Conférence du 23 avril 1911, «Évolution de la pensée allemande dans le domaine de la philosophie des sciences», p. 22 (archives : A 408/203).

19 Voir notamment les conclusions de $I R$, p. 435-436.

20 On aurait pu ajouter la loi des trois états, car Meyerson avance aussi une classification des modes d'explication qui comporte quatre états : théologique, magique, conceptuelle, mathématique (lettre à Millioud, s.d., A 408/14). Bien que Meyerson déclare que cela n'a rien à voir avec la loi des trois états de Comte, parce que ces quatre modes ne sont pas successifs dans le temps, l'inspiration comtienne est manifeste. Tout comme Gaston Milhaud en 1902, Comte brode librement sur le thème des «états» de l'esprit (G. Milhaud, Le positivisme et le progrès de l'esprit : études critiques sur Auguste Comte, Paris, Alcan, 1902), mais cette comparaison aurait surtout valeur anecdotique car la typologie des modes d'explication n'est pas une pièce maîtresse de l'œuvre de Meyerson, comme le sont la question des lois et la relation de la science au réel.

21 Comte, Cours, t. 1, leçon 1, p. 23; t. 2, leçon 51, p. 215; Discours sur l'esprit positif, ch. 7, p. 52-53.

22 Voir la lettre à Millioud (s.d., A 408/14) : «En effet, tous les phénomènes sont, en réalité, interdépendants, et chacun d'entre eux est la conséquence de 
l'ensemble de ceux qui l'ont précédé. Or un état déterminé de l'univers, à nos yeux, ne se reproduit jamais exactement, ne serait-ce que parce que la position des étoiles ne revient jamais à ce qu'elle était à un instant donné. Pour se rendre compte que ce n'est point là une réflexion purement abstraite, il suffit de se rappeler que certains Ioniens croyaient que si les planètes revenaient à une situation identique à l'égard de la terre, tous les événements terrestres devaient se reproduire, le cercle fermé que l'on parcourrait ainsi sans cesse formant ce qu'on appelait la "Grande Année". Il est clair d'ailleurs que si la connexité entre tous les phénomènes était indistincte, nous ne pourrions abstraire de règles, et par conséquent, rien prévoir, ni vivre dans le monde qui nous entoure. Si nous y parvenons, c'est qu'il y a, dans cette connexité, une sorte de hiérarchie, qui fait qu'un phénomène n'est influencé, en première ligne, que par un petit nombre de facteurs. [...] Balfour a qualifié cette particularité de notre univers de "structure fibreuse"".

23 Voir par exemple Comte, Discours, §19, p. 85-86. 